\title{
Propagation Studies Using Direction-Finding Techniques ${ }^{1,2}$
}

\section{Edgar C. Hayden}

(August 26, 1960)

The most persistent and difficult problem in radio direction finding has been measurement of the direction of arrival of an incident signal field under circumstances where multipath propagation is possible. In the $\mathrm{HF}$ band, the ionosphere plays a predominant role in the propagation of radio waves, and in this region several mechanisms exist which promote splitting of a radio signal into numerous components.

It would be of value to have a more thorough knowledge of the characteristics of the individual signal components. In this paper two techniques are described for the study of multicomponent signals. One involves use of pulse transmissions to effect "time-of-arrival" resolution; the other involves use of a highly directive antenna system to effect "directionof-arrival" resolution.

Results of the application of these two techniques in specific instances are presented.

\section{Introduction}

In the $\mathrm{HF}$ band (3 to $30 \mathrm{Mc} / \mathrm{s}$ ) long-distance transmission is possible with moderate power levels because of the existence of the ionosphere. However, while the ionosphere makes such transmission possible, it at the same time has characteristics which make difficult the measurement of signal direction of arrival and the extrapolation of such measurements for position fixing or propagation studies. The chief of these difficulties is that frequently there are in fact several possible ray paths between transmitter and receiver, and these paths do not lie exactly in the plane of the great circle passing through the transmitter and receiver locations. The former phenomenon complicates the direction-of-arrival measurement problem, and the latter the problem of extrapolation of the direction-of-arrival measurements to the point of origin of the signal.

The mechanisms giving rise to multiple paths through the ionosphere; i.e., the stratification of the region, magneto-ionic splitting, and irregularities of structure, are well known in principle, if not in details of behavior.

As a consequence of the multiple signal rays arriving at a receiving point, the field in the neighborhood of the receiving antenna exhibits "interference" or "fringe" effects. Most direction-measuring techniques give an indication which approximates the normal to the equiphase surface in the immediate neighborhood of the antenna system. In such an interference field, the equiphase surfaces are corrugated rather than plane, so that the indicated direction of arrival depends on the location of the instrument in the field. Since the field pattern moves about as the relative phases of the various rays change, the indi-

1 Contribution from Radio Direction-Finding Research Group, Department of Electrical Engineering, University of Illinois, Urbana, Ill.

2 Paper presented at the Conference on Transmission Problems Related to High-Frequency Direction Finding, at UCLA, June 21-24, 1960. cation will fluctuate. Thus, for multiray fields, the normal to the equiphase surface is not a satisfactory definition of "direction of propagation" for purposes of ray retracing.

The nature of the problem is illustrated in figure 1.

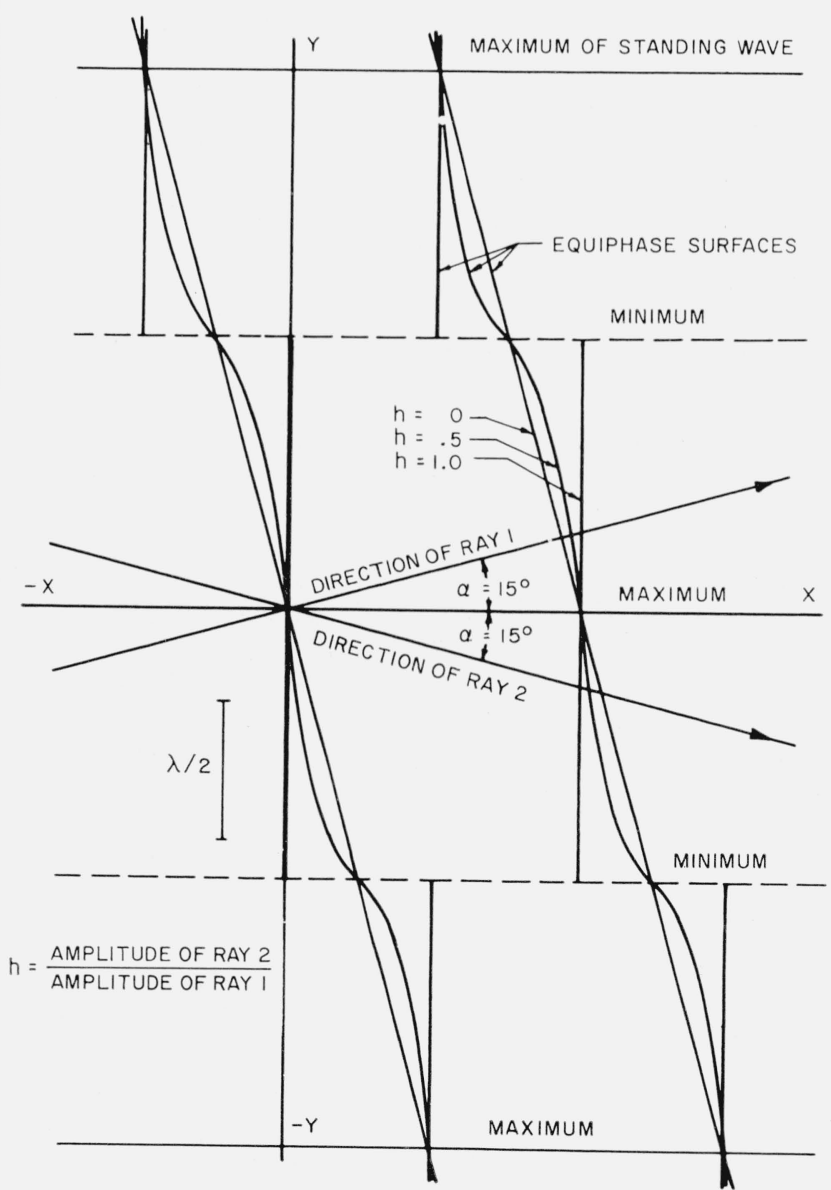

FIGURE 1. Wave interference pattern for ray separation of $30^{\circ}$. 
When two waves, each of which is assumed to be a plane wave with its wave normal lying in the plane of the paper, arrive at a receiving site from different directions, a standing wave field is produced. If (as on a transmission line) the two are going in opposite directions, the maxima and minima are each spaced one-half wavelength, and lie in planes perpendicular to the directions of propagation.

The equiphase surfaces are also planes perpendicular to the direction of propagation. If the angle between the directions of propagation of the waves is reduced from $180^{\circ}$ toward zero (it is $30^{\circ}$ in figure 1 ), the maxima and minima remain plane surfaces which are parallel to the bisector of the angle between the wave normals or rays. Their spacing, however, increases. It is

$$
s_{M-M}=s_{m-m}=\frac{\lambda}{2 \sin \alpha}
$$

$s_{M-M}$ is the distance between maxima

$$
s_{m-m} \text { is the distance between minima }
$$

\section{$\lambda$ is the wavelength} $2 \alpha$ is the angle between the wave normals
or rays

Unlike the maxima and minima, the equiphase surfaces do not remain plane. They become corrugated (or, if the two waves are of exactly equal strength, broken). "On the average," they are perpendicular to the wave normal of the stronger ray. If the waves are assumed to be in phase at the origin, the intersections of the equiphase surfaces with the $X Y$ plane (the plane of the paper) are given by the following expression:

$$
\begin{aligned}
Y & =-\frac{\lambda}{2 \pi \sin \alpha} \arctan \left[\frac{1+h}{1-h} \tan \frac{(2 \pi X \cos \alpha)}{\lambda}\right]-\frac{n \lambda}{\sin \alpha} \\
n & =\ldots-3,-2,-1,0,+1,+2, \ldots
\end{aligned}
$$

It can be seen in figure 1 that the normal to the phase front varies from a direction between the wave normals (but closer to the normal to the stronger wave), to a direction outside the angle between the wave normals (but away from the normal to the weaker wave). Thus, as the relative phase between the waves changes, the normal to the equiphase surface at a fixed point will swing back and forth, across the direction of the normal to the stronger wave. As the two signals approach equality in strength, the swing approaches $90^{\circ}$.

When the angle between the wave normals $(2 \alpha)$ is fairly small, the distance between the corrugations of the phase surfaces is several times as great as the dimensions of a small aperture system. For example, if $\alpha$ is $5^{\circ}$, the distance between adjacent corrugations is over $5.5 \lambda$. This is about 22 times the maximum useful size ${ }^{3}$ of a 4-element Adcock system, and about 5.5 times the maximum useful size ${ }^{3}$ of a regularly spaced 8-element Adcock system. All present small aperture direction-finding systems give an indicated bearing which is equal to, or very nearly equal to, the normal to the equiphase surface (this is the commonly used definition of the direction of propagation). Thus these small systems can give directional indications which are considerably (up to $90^{\circ}$ in the extreme case) in error. The indicated bearing can show considerable fluctuation even though the actual ray arrival angles are steady.

In figure 2 the error in indicated bearing is shown graphically for the particular case in which $2 \alpha$ is $30^{\circ}$. Curves are given for several values of the relative amplitude of the waves. The error curves for other values of $\alpha$ are similar in character. Note that as the relative phase varies, the deviation to one side of the stronger ray is less than that to the other side.

Similar effects occur in systems using arrays of large aperture, though the error fluctuations can in general be confined to a smaller range. They may, however, be considerably greater than the true fluctuations in direction of arrival of the rays.

Thus, under ordinary circumstances, directionfinding systems of both large and small aperture are subject to errors in bearing indication when the incident signal is multirayed. If, then, the characteristics of an individual ray are to be studied, some means must be provided for ray separation; i.e., for taking the signal apart into its components. Three possibilities are immediately apparent:

(1) Wait until only one ray is present before acquiring data.

(2) Take advantage of differences in ray path length to effect a resolution on the basis of time of arrival.

(3) Take advantage of differences in ray path direction to effect a separation on the basis of direction of arrival.

The first of these very severely restricts the situations under which data can be acquired. In practice it is of limited utility. The second requires the use of signals consisting of short pulses, the resolution capability being determined by the pulse duration. The third requires large antenna systems of high directivity. The resolution capability is limited by the beamwidth of the array radiation pattern.

The latter two of the techniques listed are both useful. Each covers some situations in which the other fails. In particular, the time-of-arrival technique is often satisfactory for resolution of the normal modes of propagation, but fails under anomalous conditions because of the great multiplicity of raylets and the extremely short pulse duration which would be required for resolution. On the other hand, the

${ }_{3}$ The maximum useful size of these systems is determined by the maximum permissible spacing error. The figures above are for systems whose maximum spacing error is limited to about $2^{\circ}$. 


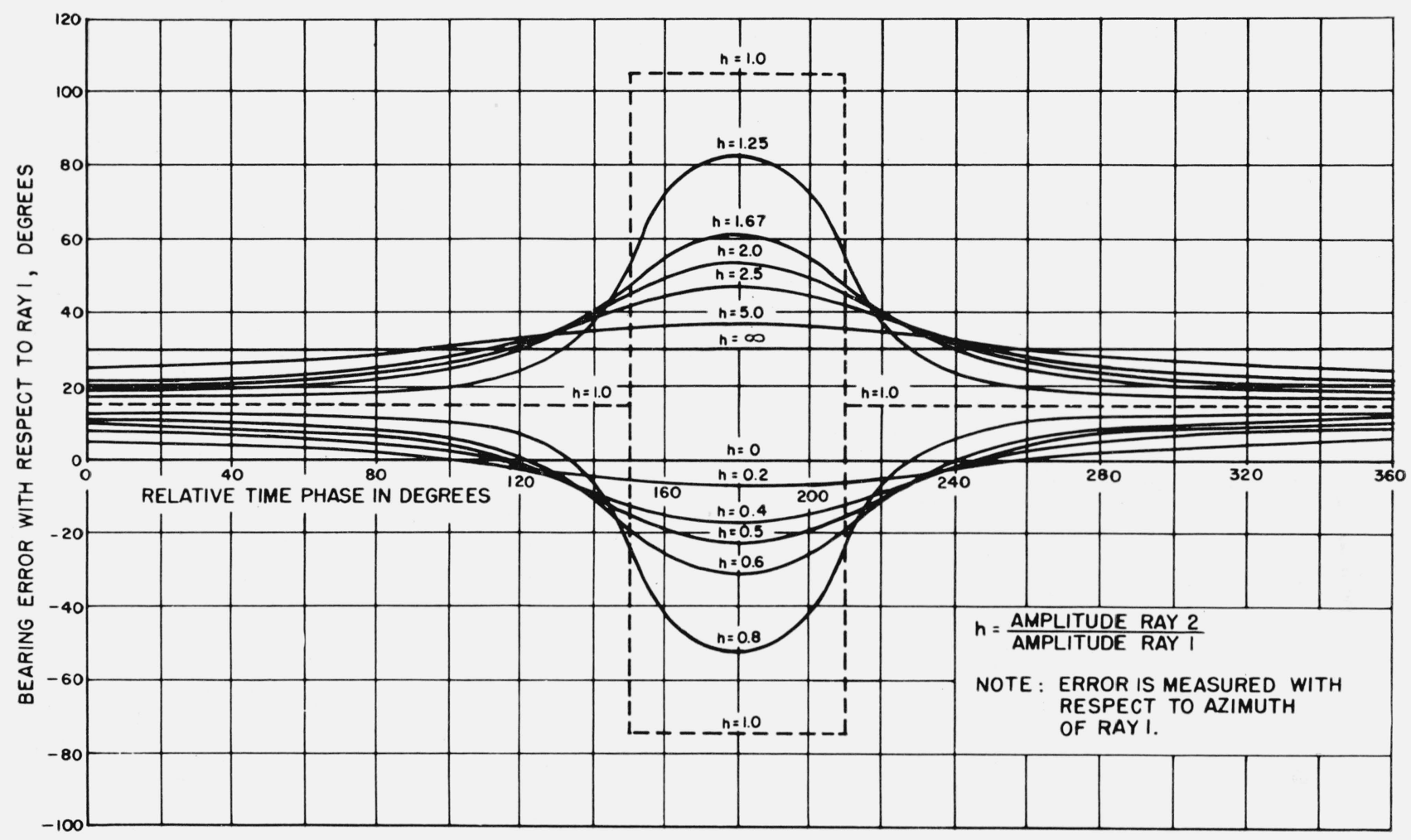

Figure 2. Wave interference bearing error for two-ray signal with ray separation of $30^{\circ}$.

direction-of-arrival technique can often give useful information under anomalous conditions because the angular spectrum of such signals is frequently of large extent, but may fail under normal conditions because the angular separation of the rays is small. Some of the advantages of each technique have been exploited. The results of two specific experiments are reported in the following sections.

\section{Ray Resolution by Time-of-Arrival}

The technique used in this experiment for taking apart a multiray signal involves exploitation of the differences in time of arrival of the various rays. Such a technique, while not suited to the general run of signals, is well-suited for use under controlled circumstances. With a cooperative transmitter, the signal modulation characteristic can be chosen to facilitate the receiving problem.

For resolution of multiray signals a short pulse is required. Since direction-finding techniques must often be used with transmissions which are modulated by waveforms other than pulses, it would be useful to provide a signal to simulate other sorts of transmissions as well. Comparisons would thus be facilitated between measurements of the directional characteristics of the component modes, and the directional indications given on the composite signal by various instruments. Such a signal can be provided by transmitting alternately a pulse short enough to resolve the component modes and a pulse long enough that the received pulses for the various modes overlap an appropriate amount.

Choice of a length for the short pulse is a compromise between the resolution required on one hand, and signal-to-noise ratio, available receiver bandwidth, Federal Communications Commission regulations, and the overcrowded state of the $\mathrm{HF}$ band on the other hand. The latter two considerations seemed to be the deciding factors. In order to avoid interference from other transmitters on adjacent channels, and to avoid interference with other users of the spectrum, the bandwidth was limited to $25 \mathrm{kc} / \mathrm{s}$. For double-sideband transmission the minimum usable pulse width under these conditions is about $80 \mu \mathrm{sec}$. Fortunately this was short enough, for the transmitter location chosen, to resolve the grossly different modes, though it was only occasionally that the two magneto-ionic components of a given mode could be resolved completely.

The greatest expected difference in time of arrival between modes of any consequence was of the order of $4 \mathrm{msec}$. A long pulse of $12 \mathrm{msec}$ duration was considered adequate to insure overlap of all interesting modes, and to provide a composite signal of sufficient duration.

The modulation waveform chosen for the transmitted signal is shown in figure $3 \mathrm{a}$. If a signal of this configuration were to travel to a receiving location via two paths, the structure of the received signal 

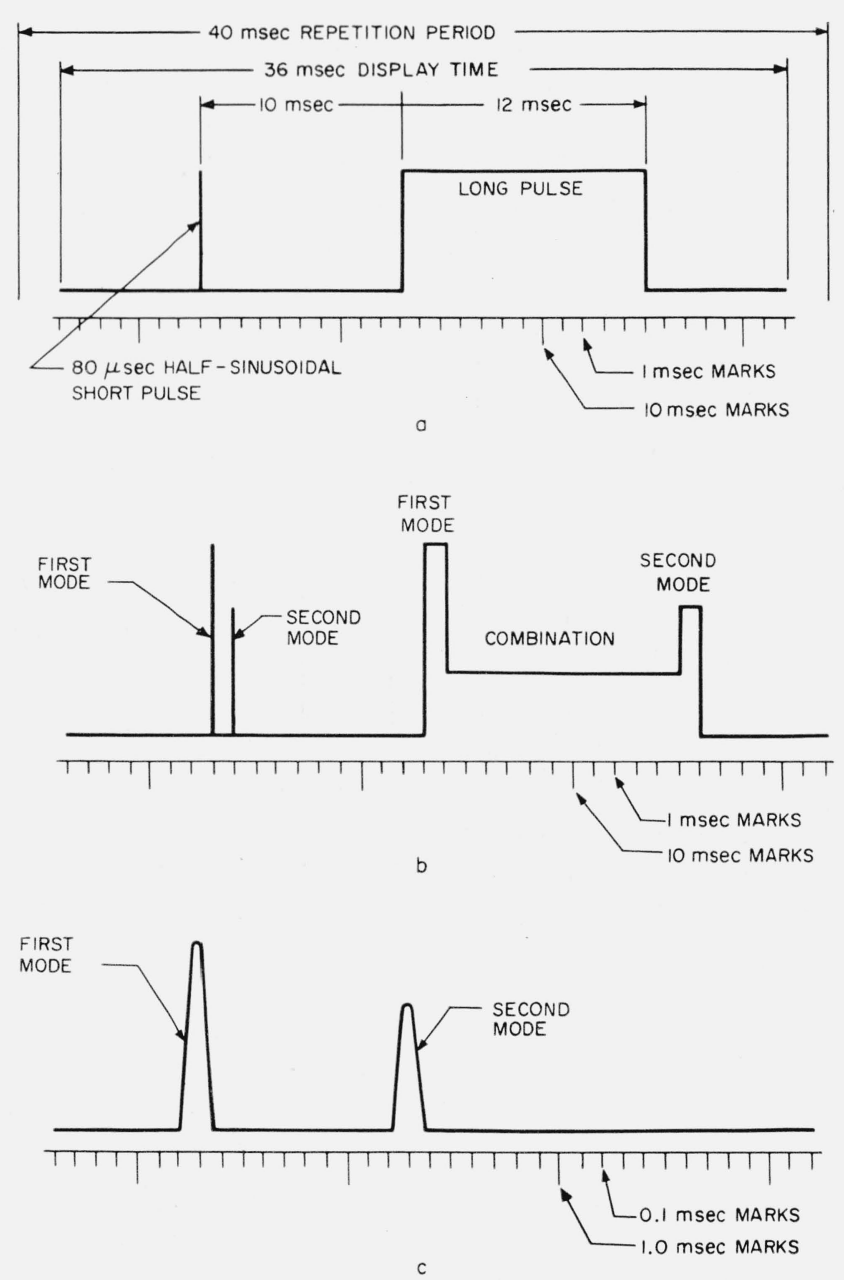

Figure 3. Signal envelope waveforms.

$$
\text { a. Transmitted waveform. }
$$

b. Example of a received waveform; two modes, amplitude of first $3 / 2$ that of second, relative phase $180^{\circ}$, difference in arrival time $1 \mathrm{msec}$.

c. Expanded sweep, short pulses only.

might, for example, be as shown in figure 3b. The long pulse shows three regions, a leading edge in which only the first-to-arrive ray is present, a trailing edge in which only the last-to-arrive ray is present, and a central region in which a composite of the rays is present. Thus the long pulse permits some decomposition of the signal, but also permits study of the composite signal. If the difference in time of flight for the two rays is more than the duration of the short pulse, then two distinct short pulses will be observed (fig. 3c). In some cases each of these short pulses can be further split into magneto-ionic components, thus allowing an additional step in the decomposition of the signal.

Because of its ability to work on pulse signals, the directional measurement system chosen was a conventional Adcock antenna system followed by a twin-channel receiver and a cathode-ray-tube goniometer. The elements of the system are shown in figure 4.

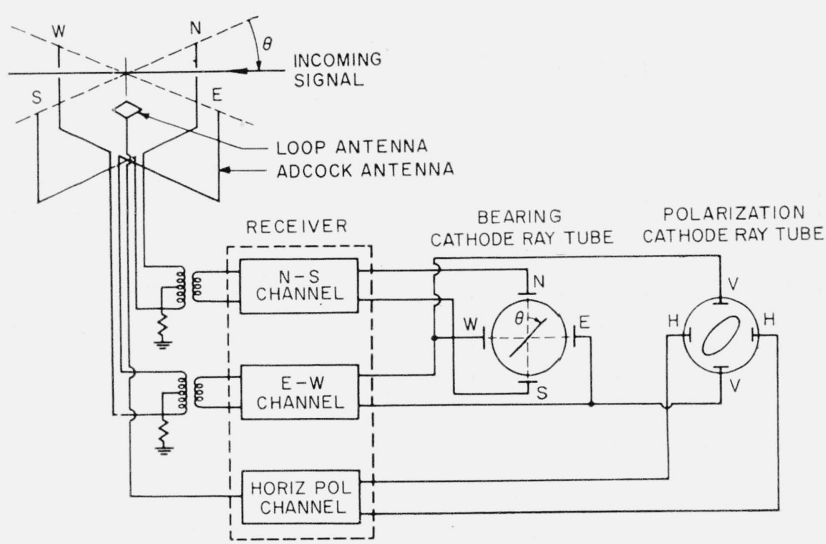

Figure 4. Direction-finding system, block diagram.

In addition to the bearing, information about the state of polarization was needed. A horizontal loop antenna was installed in the center of the antenna array. It can be seen in figure 4 . Its output was received by a third channel of the direction-finding receiver. Since the direction-finding antenna was vertically polarized, and since signals were expected to come from an easterly direction, the output of the $E-W$ channel of the direction-finding receiver was used, with the output from the loop receiver, to display the state of polarization on a cathode ray tube. Quantitative measurement of the state of polarization was not needed, so this simple system sufficed in spite of the different directional characteristics of the horizontally and vertically polarized parts of the system.

Three cathode ray tubes, as shown diagrammatically in figure 5, were used in the display system, one to display bearing, a second polarization, and the third signal structure. The third tube was a two-gun tube, one gun being used to display the vertically polarized signal, and the other the horizontally polarized signal. In addition, each gun was double-traced, alternate traces serving to display signal structure and timing scale. A mode selection gating pulse, variable in

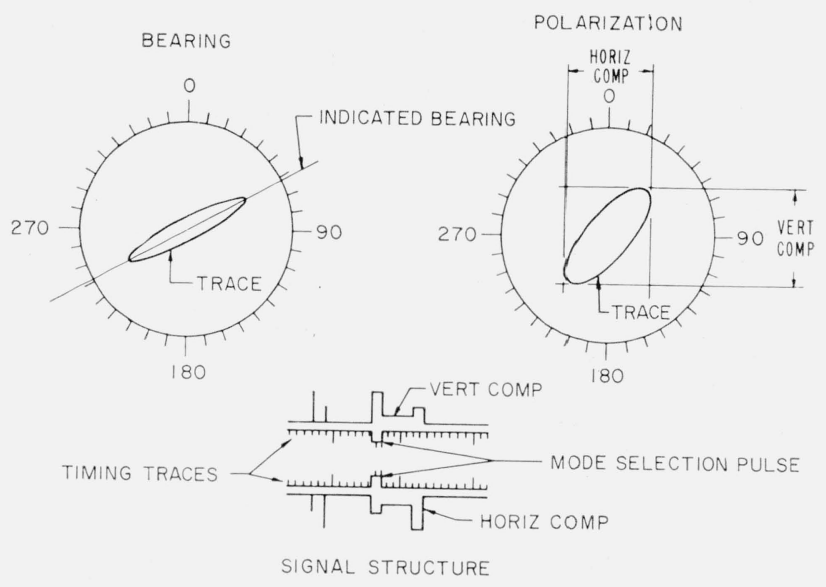

Figure 5. Key to interpretation of signal display. 
duration and in position along the structure trace, was provided to select portions of the signal for display on the bearing and polarization indicators. The duration and position of this gate are shown as a "step" in the timing scale base line. In some cases an expanded structure sweep was used, showing only the short pulses as illustrated in figure $3 \mathrm{c}$. The expansion factor is roughly $10 \mathrm{X}$.

Transmitter pulse repetition rate and receiver display rate were synchronized by deriving each of them from a stable $100 \mathrm{kc} / \mathrm{s}$ oscillator. The basic pulse repetition rate was $25 / \mathrm{sec}$.

The displayed information was recorded with a specially constructed pulse-driven $16-\mathrm{mm}$ camera, whose operation was controlled by the timing pulse train. The next few figures show examples of typical signal displays. The figures were made from individual frames of the actual $16-\mathrm{mm}$ film recording. Reference to figures 3 and 5 will aid in understanding figures $6,7,8$, and 9 . In the figures showing the complete signal waveform, the camera shutter was open for one data cycle $(2 / 25 \mathrm{sec}$ because of the double-tracing of the structure display to present both timing and data traces). The shutter was opened and closed in the sweep flyback periods. Thus one timing and signal structure trace, but two successive bearing and polarization traces, were recorded. In the figures showing the short pulses only, the shutter was open for five complete data cycles $(10 / 25 \mathrm{sec})$, causing five timing and signal structure traces, but ten bearing and polarization traces, to be recorded. The multiple exposure of the short pulse signals was of material help in reducing the effects of noise on the bearing display.

Figure 6 shows a two-mode signal (one-hop $E$ and one-hop $F$ ). The two short pulses are visible near the left end of the structure display traces. The long pulse shows three portions, a portion where only the first mode is present, a portion of composite signal, and a portion where only the second mode is present. The mode selection pulse is set to select the first mode and a portion of the composite signal. Bearings and polarization ellipses for these two portions of the signal appear superposed on the appropriate indicators. The two superposed images on each of the indicators may be differentiated by comparison of their relative dimensions with the relative amplitudes of the signal components as shown on the structure display. Note that only the vertically polarized (upper) component of the structure display is of use in interpreting the bearing display, since the direction-finding antenna was vertically polarized. Both components of the structure display are useful in interpreting the polarization display. This series of five pictures shows one fading cycle, as the relative phase of the components takes on successive values of approximately $0^{\circ}, 90^{\circ}, 180^{\circ}, 270^{\circ}$, and $360^{\circ}$. The steadiness of the bearing and polarization indications on the single mode should be contrasted with the variability of these indications on the composite portion of the signal. The behavior of the bearing indication on the composite portion of the signal should be compared with the wave interference bearing error curves in figure 2 .

Figure 7 shows a similar fading cycle, but the mode selection pulse is set to pick out the second mode, rather than the first, and a portion of the composite signal.

Figures 8 and 9 show a one-hop $F$-layer signal. The structure trace is in the "fast sweep" position so that only the short pulses are displayed.

In figure 8a only the ordinary magneto-ionic component is present, while in $8 \mathrm{~b}$ only the extraordinary component is present. These two can be differentiated by their characteristic polarizations, which are quite stable from day to day. Note the difference in indicated bearing. In figure $8 \mathrm{c}$ both components are present, and are nearly resolved. Both characteristic polarizations are evident. The bearing display is not easily interpretable because the bearings of the two components are so nearly alike that the indications are not distinct.

Figure 9a shows a case in which both magnetoionic components are present, but are incompletely resolved. In the polarization display the figure starts to build up in one of the characteristic polarizations, then switches to the other. During the transition, or overlap, the vertically polarized components are in phase, but the horizontally polarized components are out of phase. This causes the vertical hourglass shape of the polarization display and propeller shape of the bearing display. This also shows up in the splitting of the horizontally polarized pulse, but not of the vertically polarized pulse. The bearing trace is in effect, swinging from the bearing of one component to that of the other through a small angle. In figure $9 \mathrm{~b}$, the situation is similar, except that the vertical components are out of phase and the horizontal components are in phase. In this case the hourglass figure on the polarization display is horizontal. The vertically polarized pulse is split, but the horizontally polarized one is not. The bearing trace swings from the bearing of one component to that of the other through an angle of nearly $180^{\circ}$, giving the effect of a split bearing. The wave interference bearing error curves (fig. 2) will assist in interpreting the bearing displays in figures $9 \mathrm{a}$ and $9 \mathrm{~b}$. Note in figure 2 that if two components are in phase, and their relative amplitude swings from zero to infinity, the bearing will swing from that for one signal to that for the other through a small angle, while if the components are out of phase, the bearing will swing through the supplement of this angle. It is this action that is taking place in figures $9 \mathrm{a}$ and $9 \mathrm{~b}$. Figure $9 \mathrm{c}$ shows a case where the overlap is so nearly complete that neither the horizontally nor the vertically polarized pulses, are split. However, the presence of the two components can still be detected by the $X$-shaped figure in the center of the polarization display (compare with figs. 8a and $8 \mathrm{~b}$ in which only one component was present).

The results reported here are for a particular single path, and a particular frequency. Though 
they are for this reason somewhat specialized, they do show some of the phenomena that are observable, and do demonstrate the power of the technique. The transmitter was located at Columbus, Ohio, and the receiver at Urbana, Illinois. Both stations lie within a few minutes of the 40th parallel of latitude. They are separated by a distance of $450 \mathrm{~km}$. The frequency used was $5155 \mathrm{kc} / \mathrm{s}$. The transmitter delivered a peak power of 600 watts to a horizontal dipole whose axis had a north-south orientation. The great circle bearing of the transmitter at the receiver was $88 \frac{1}{3}$ degrees. Data were taken only during the hours between evening twilight and midnight.
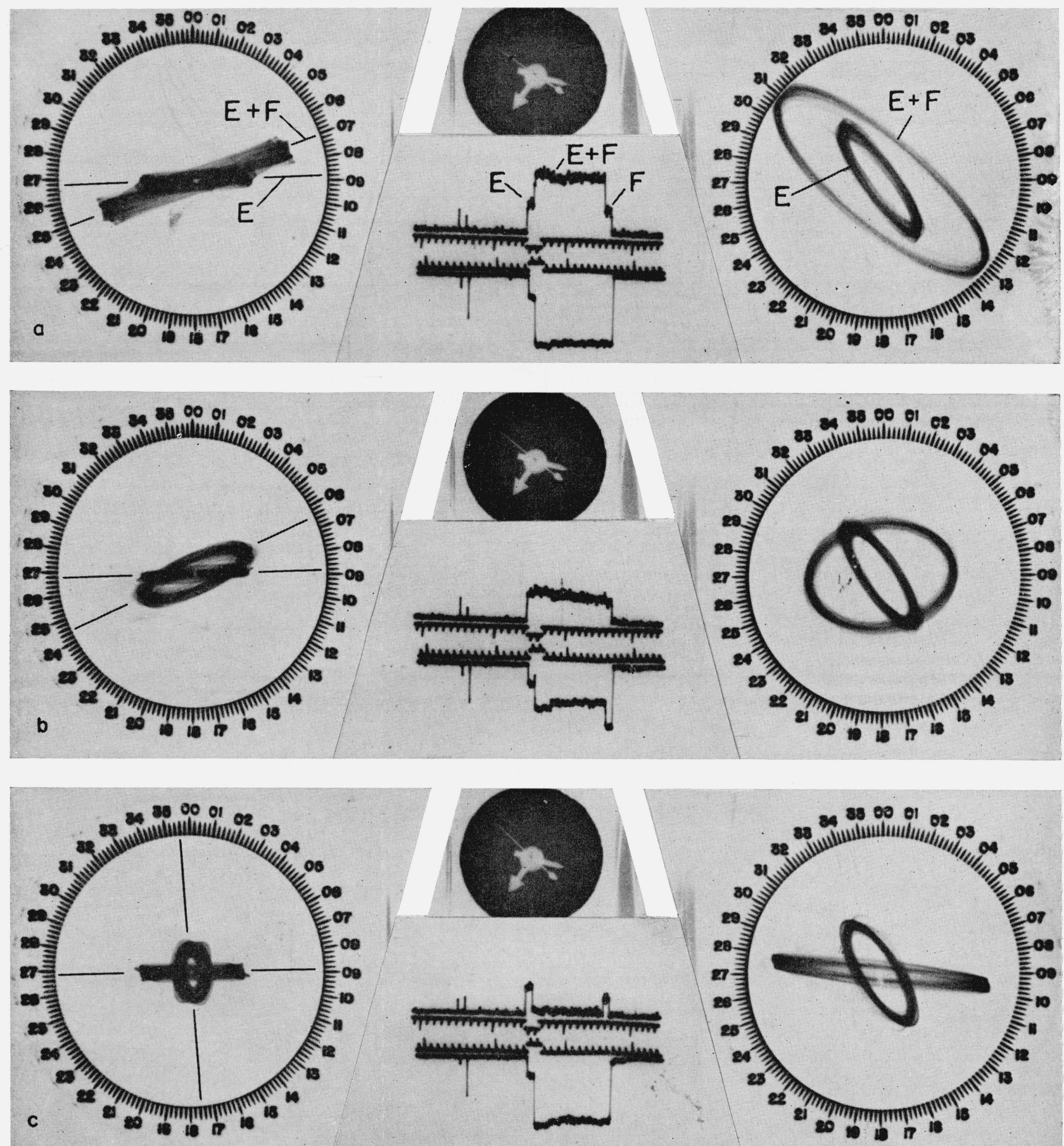

Figure 6. Typical composite signal with one-hop $E$ and one-hop $F$ modes, one-hop E and composite selected for presentation on bearing and polarization displays; successive frames illustrate slip in phase $(\phi)$ between rays over one fading cycle. 

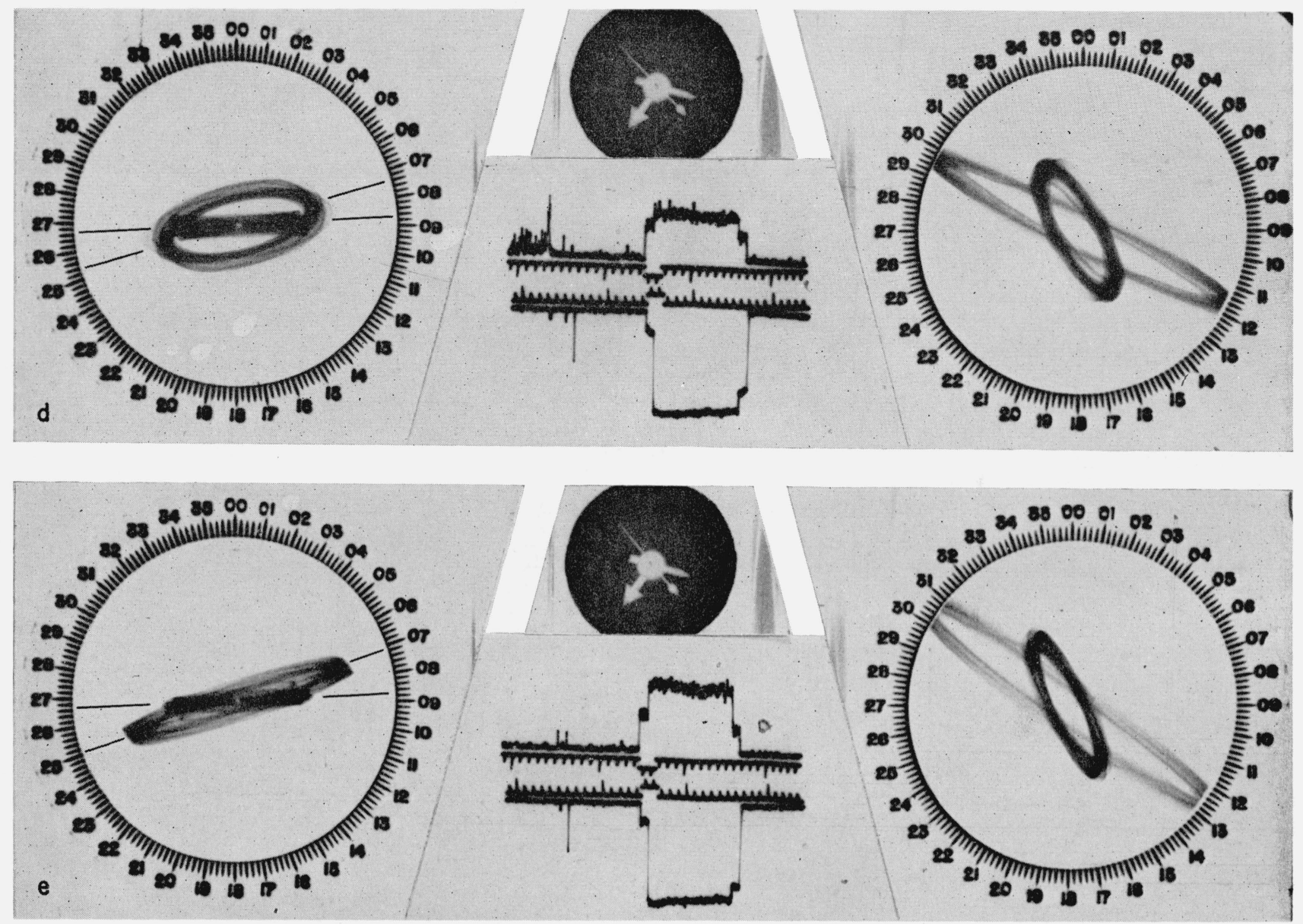

Figure 6. Typical composite signal with one-hop $E$ and one-hop $F$ modes, one-hop $E$ and composite selected for presentation on bearing and polarization displays; successive frames illustrate slip in phase $(\phi)$ between rays over one fading cycle-Cont.

d, $\phi=270^{\circ} ; \mathrm{e}, \phi=360^{\circ}$.

At the times the data were acquired, the observed MUF for this path for one-hop F-layer transmission was above the operating frequency during the entire daily transmission period. The MUF for normal one-hop E-layer transmission dropped below the operating frequency shortly after the beginning of the transmission period. Mode identification was accomplished by continuously observing the signal structure pattern throughout the entire late afternoon and evening, from the time when both the normal one-hop $E$ - and $F$-layer modes were present, through the period of breakup of the normal E-layer mode. The polarization states characteristic of the two magneto-ionic components of the one-hop $F$-layer mode were determined by observing the polarization display generated by the short pulse through that period where the two components interchanged order of arrival as the normal E-layer breakup occurred. Only on rare occasions was appreciable splitting of the E-layer mode observed.

The significant observed transmission modes are shown in terms of relative time of arrival in figure
10. The times of arrival are all referenced to the one-hop $F$-layer mode (designated $\mathrm{C}$ ), since that was the only mode regularly present, and always identifiable. No means was available for reference to the time of transmission. In addition to the normal one-hop $F$-layer mode, transmission by a one-hop nighttime $E$-layer mode (designated A) was observed during most evenings, for varying periods of time. In fact all the data recorded on E-region signals was obtained on such sporadic transmissions. The mode designated $B$ was tentatively identified as a one-hop reflection from the $F_{1}$ region. Its relative time of arrival suits that situation, and its direction of arrival was essentially on-path. The other modes were not positively identified because their occurrence was not sufficiently frequent or prolonged to permit many observations to be made. However, the mode designated $F$ was probably a 2 -hop $F$-layer reflection.

Bearing information was read from the film recordings, frame by frame, and punched onto paper tape for analysis by the Illiac, the University of Illinois' electronic digital computer. Well over 12,000 in- 

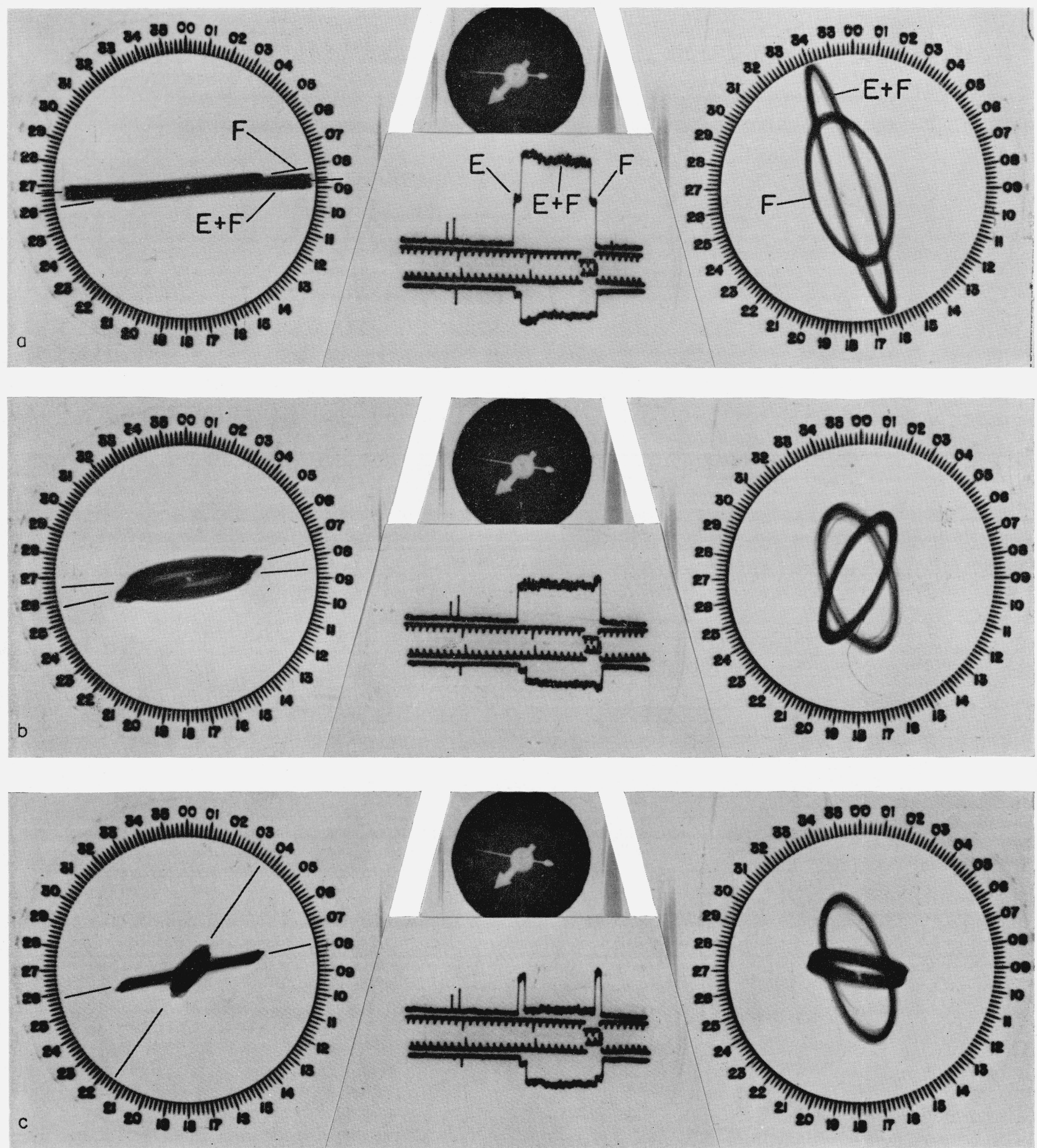

FiguRE 7. Typical composite signal with one-hop $E$ and one-hop $F$ modes, one-hop $F$ and composite selected for presentation on bearing and polarization displays; successive frames illustrate slip in phase $(\phi)$ between rays over one fading cycle. a, $\phi=0^{\circ} ; \mathrm{b}, \phi=90^{\circ} ; \mathrm{c}, \phi=180^{\circ}$. 

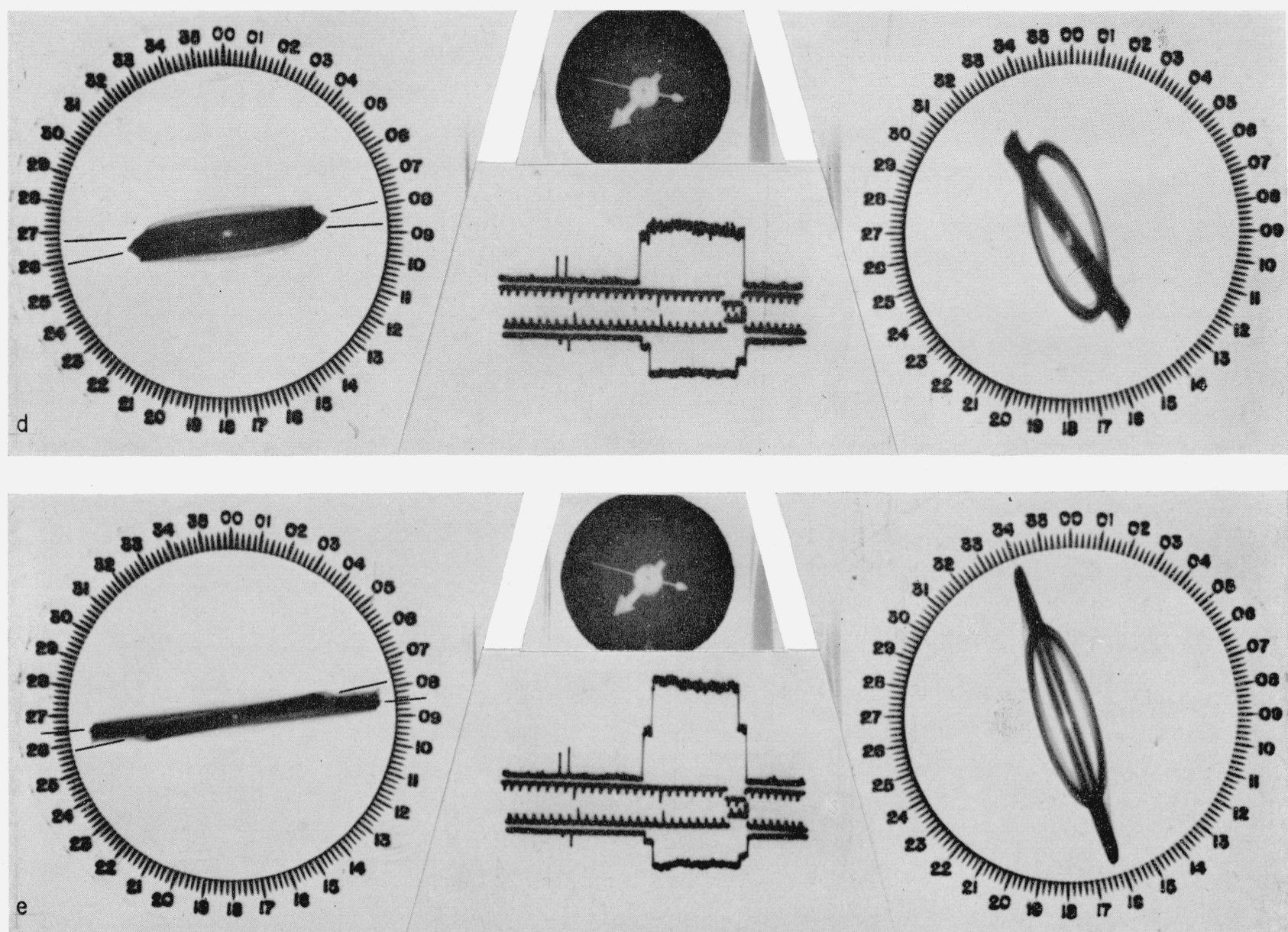

Figure 7. Typical composite signal with one-hop $E$ and one-hop $F$ modes, one-hop $F$ and composite selected for presentation on bearing and polarization displays; successive frames illustrate slip in phase $(\phi)$ between rays over one fading cycle-Cont.

$$
\mathrm{d}, \phi=270^{\circ} ; \mathrm{e}, \phi=360^{\circ} \text {. }
$$

dividual frames were read. Each sample bearing determination consisted of readings or "snaps" from a series of frames, ranging from 10 to 286, and averaging about 40. Figure 11 gives examples of bearing samples of various types. The range of variability, classed by propagation mode, of the snaps within a sample is shown in figure 12 . This is a measure of the rapid (second to second) fluctuation range. Information is given for one-hop propagation via the nighttime $E$-layer, the $F$-layer with ordinary and extraordinary components both present, the $F$-layer with ordinary only, and the $F$-layer with extraordinary only. On the graph bars are marked the minimum, mean, and maximum values of the RMS deviations from the sample mean of the snaps within a sample.

Of interest is the relatively high short-time directional stability of the nighttime E-layer mode. This was true in spite of the fact that both magnetoionic rays were present. They were invariably too nearly coincident to resolve. This stability seems indicative of a nighttime E-region having structural regularity over a considerable area. The structure must be such that it produces a very nearly specular reflection, rather than a structure which is patchy, thus producing scattered, or nonspecular, reflections. The rather sudden appearance and disappearance of the E-layer signal indicate relatively rapid changes in the E-layer ion density or height, causing rapid changes in the "skip" distance. Since vertical soundings show little variation in the height of reflection from the $E$-region, ion density variations, having structural uniformity over a fairly large area, seem the more probable explanation.

The large variability in the undifferentiated $F$-layer mode, compared to the single magnetoionic components of that mode, is almost surely due to wave-interference effects (resulting in corrugated phase fronts) between the two magnetoionic rays. The paths traveled by the two magnetoionic rays differ considerably more for $F$-layer transmission than for E-layer transmission. This illus- 

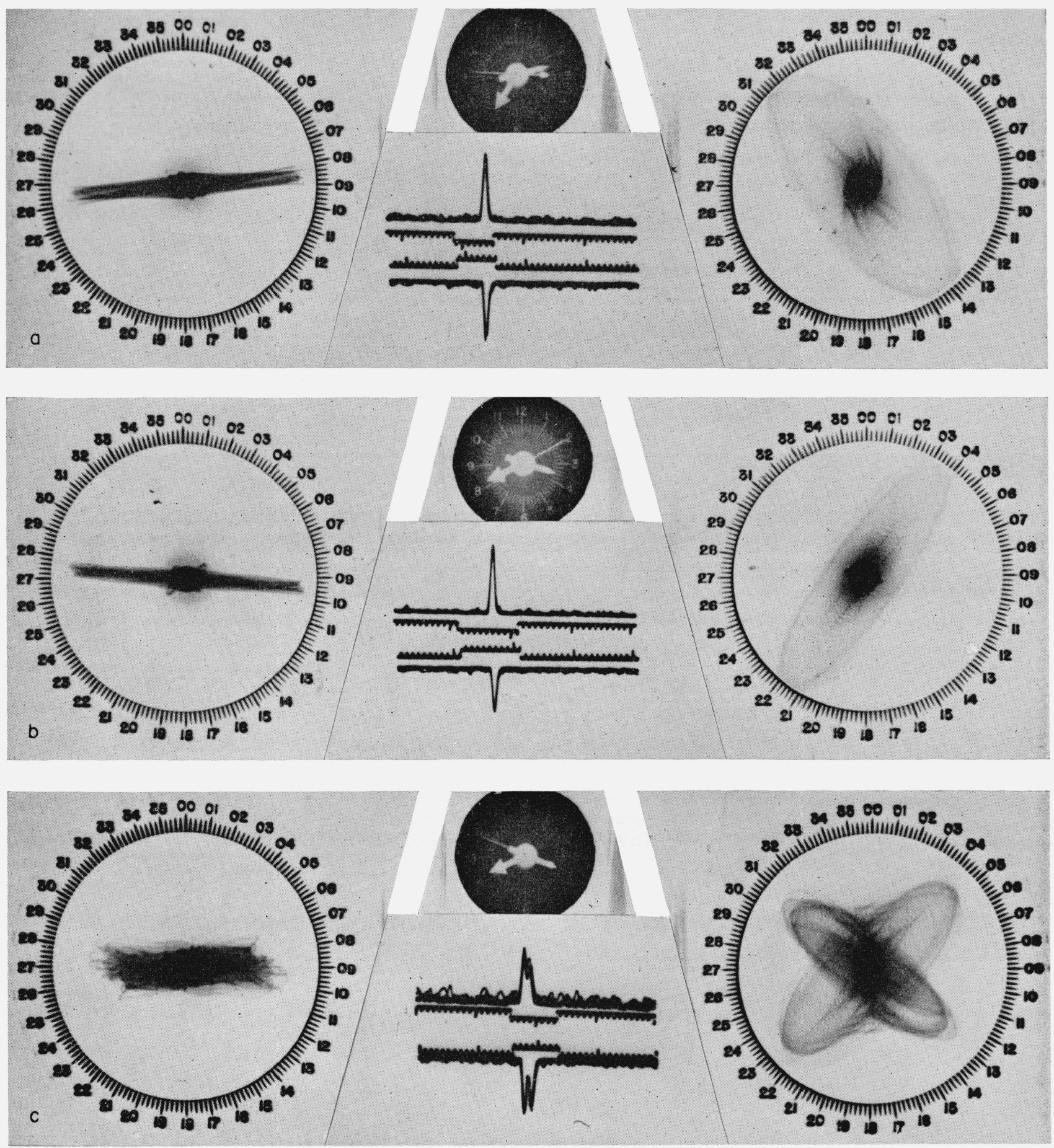

Figure 8. One-hop F mode, short pulses only, expanded sweep.

a, Ordinary ray only; b, Extraordinary ray only; c, Both rays, essentially resolved. 

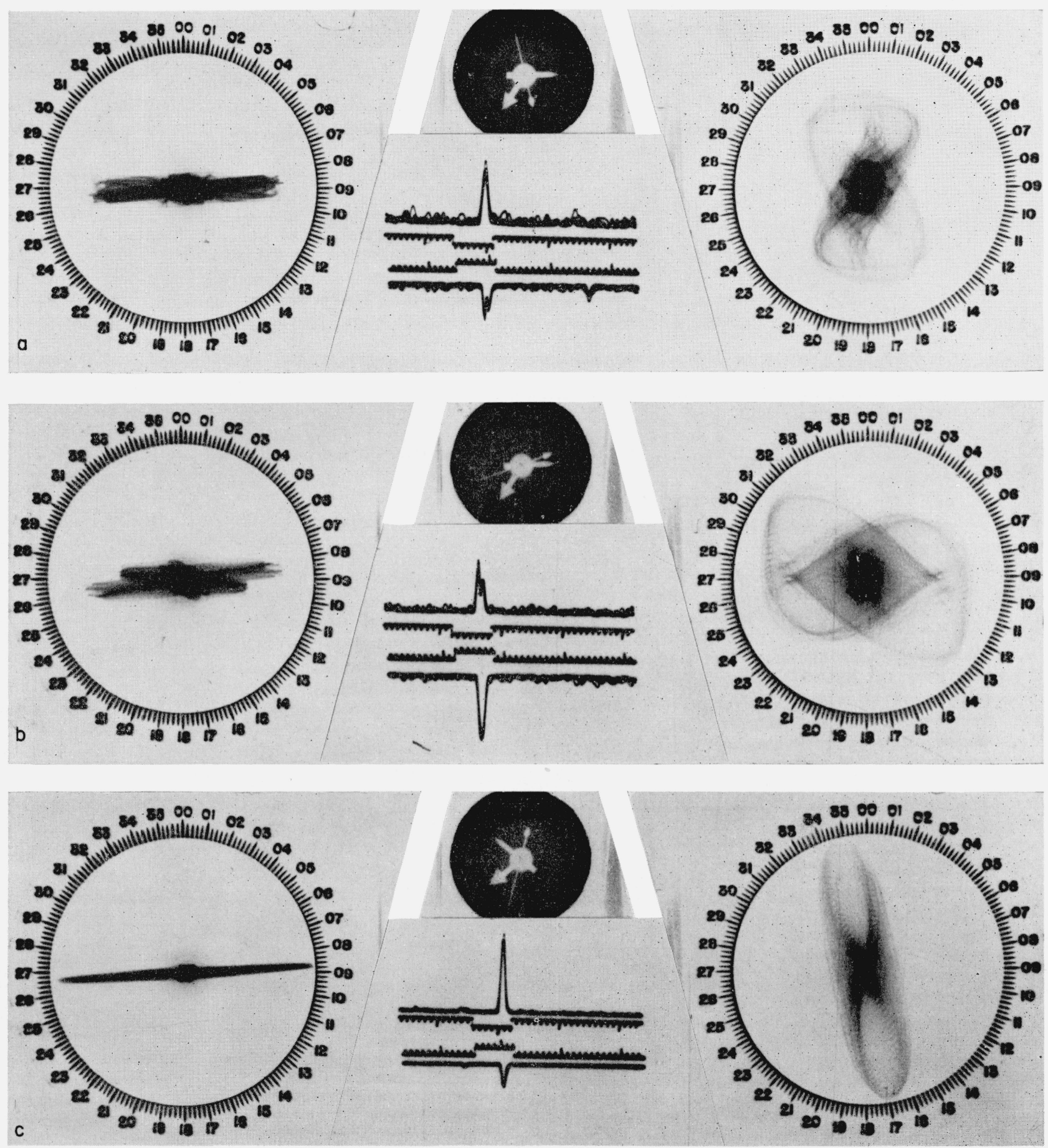

Figure 9. One-hop $F$ mode, short pulses only, expanded sweep, ordinary and extraordinary rays both present, partially resolved. a, Vertical components in phase, horizontal out of phase; $b$, Vertical components out of phase, horizontal in phase; c, Vertical components in phase, horizontal out of phase; two rays nearly coincident. 


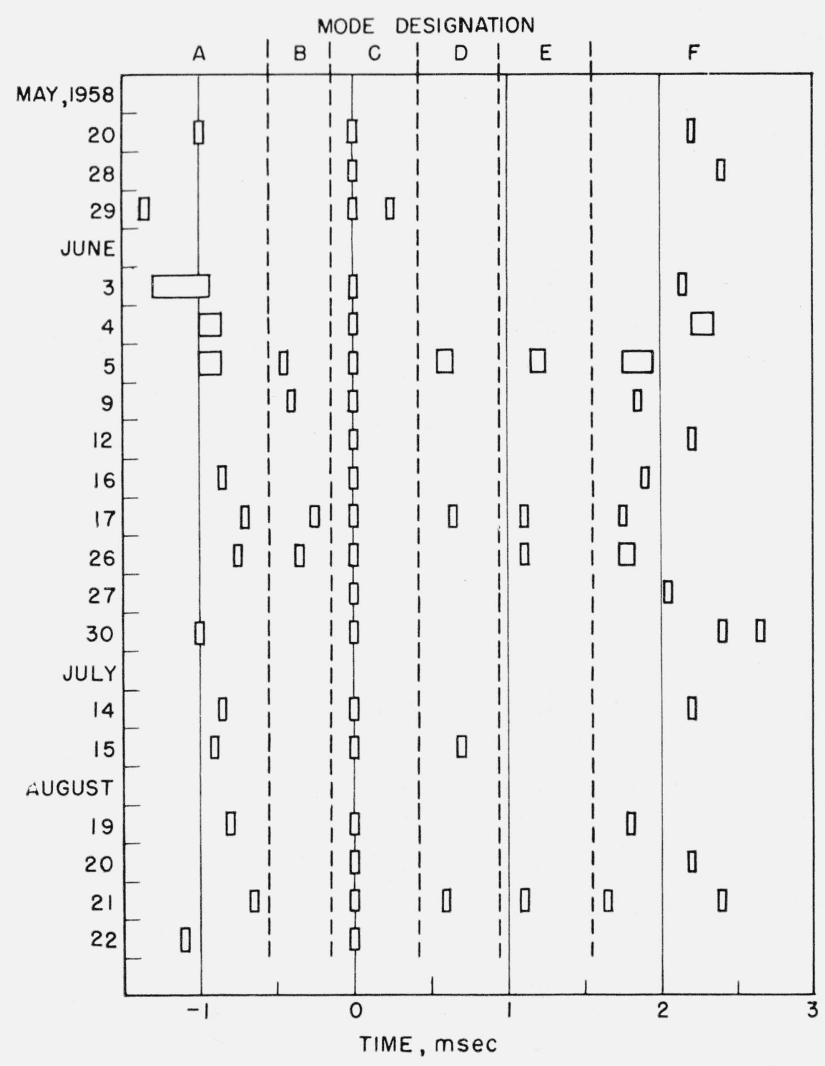

MODE A: I-HOP E NOTE: I msec IS EQUIVALENT TO $300 \mathrm{~km}$ MODE B: I-HOP $F_{1}$ NOTE: OF PATH LENGTH.

FIGURE 10. Observed mode structure: note that on a given day not all modes observed were necessarily present simultaneously. trates the importance of completely decomposing the signal to its basic rays before drawing conclusions about ray directional characteristics from the variability of the bearing indication.

Distributions of the sample means about the great circle path, taken over the entire duration of the experiment, are shown in figure 13. Notice how tightly the E-layer signals cluster about the great circle path. The long-time mean for this mode is only $0.6^{\circ}$ from the great circle bearing, and the RMS deviation of the sample means about this long-time mean is only $1.6^{\circ}$.

The $F$-layer bearing distribution chart shows an interesting split near the great circle bearing value. The reason for it can be seen in the distribution graphs for the ordinary and extraordinary components separately. The ordinary component shows a definite bias to the north. In fact, no samples in this mode showed means south of the great circle path. The extraordinary component, on the other hand, shows a definite bias to the south of the great circle path. There are a few entries to the north of this value, but examination of the data in detail reveals that these instances accompany unusually large deviations of the ordinary component to the north. Thus, at these times, both components showed an unusual northerly shift. The Basic Radio Propagation Prediction charts issued monthly by the National Bureau of Standards indicated that at these times a horizontal gradient of ion density, increasing to the north, might have been expected.

Examination of the phase refractive index of the ionospheric region shows that the sense of the bias

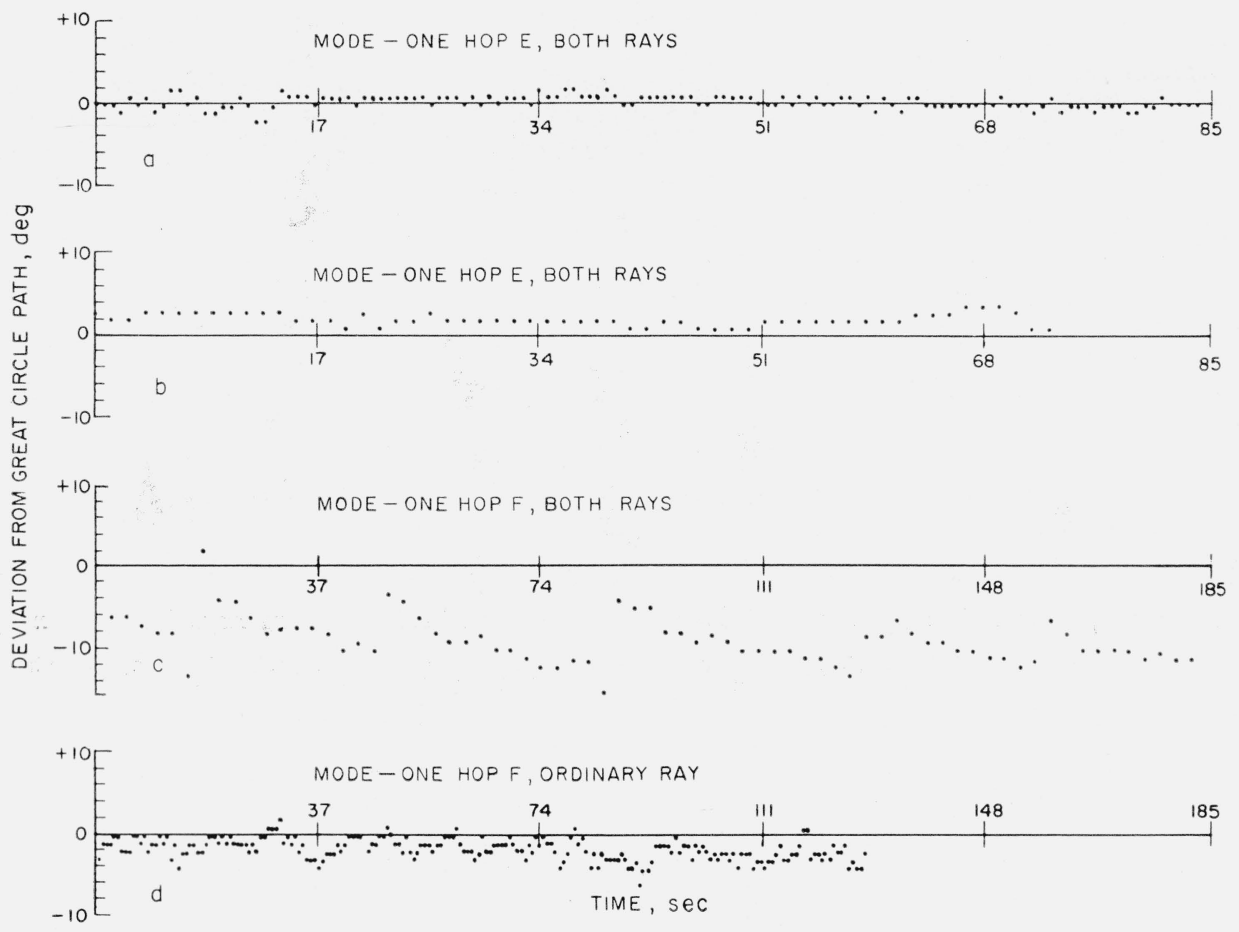

FIGURE 11. Examples of bearing samples; each sample contains a number of snaps, each snap being read from one frame of film. 


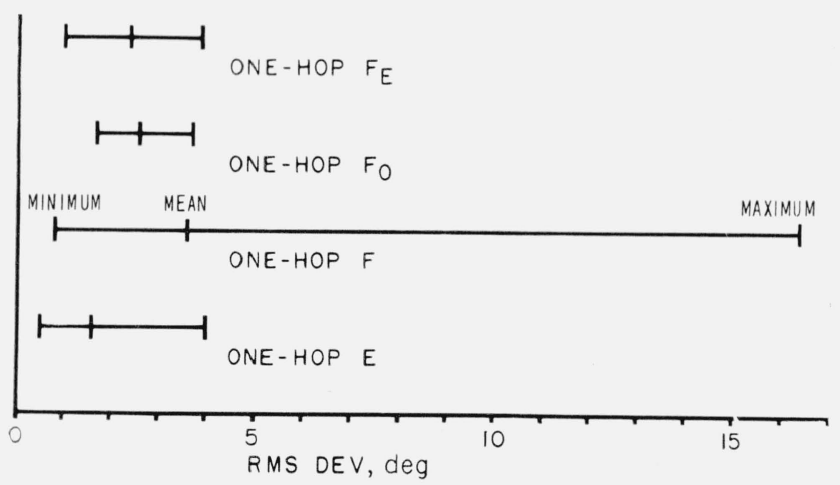

FIGURE 12. RMS variability of snaps within a sample (shorttime variability), classified by mode.

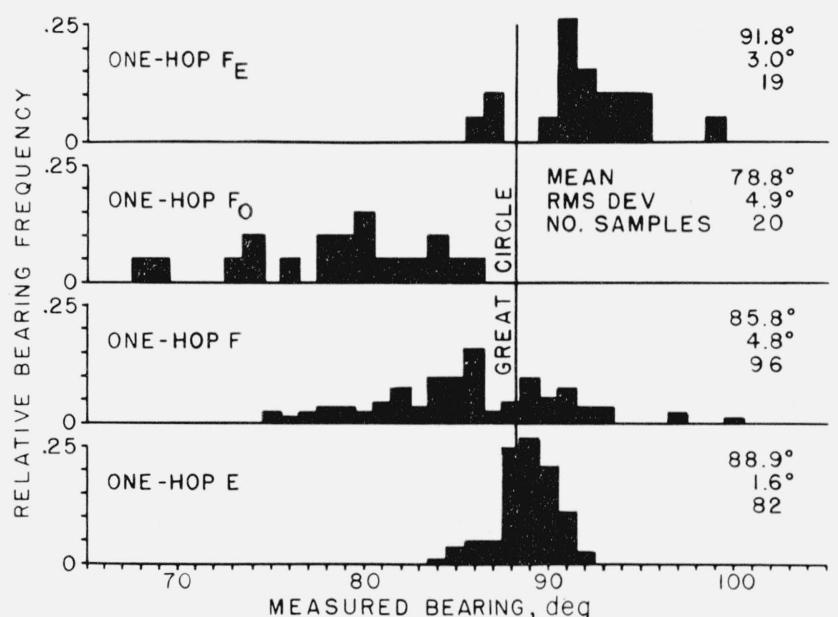

FIGURE 13. Distribution of sample means about great circle bearing (longtime variability), classified by mode.

of the two components is as should be expected. No attempt to check theoretically the magnitude of the bias has been made. Notice that the biases of the two components are not equal, the ordinary component bias averaging about $9^{\circ}$, and the extraordinary component bias averaging about $4^{\circ}$. This would be expected as a consequence of the tilt of the earth's field at this latitude. Note also that the average of the means of the ordinary and extraordinary components taken separately is only $0.5^{\circ}$ from the mean for the undifferentiated mode (the data for the two magneto-ionic components separately is not included in that for the composite mode). Thus if directional measurements on signals in this mode were taken over a period of time, they would show a northward bias. If a position fixing task permitted long-time measurements, such a bias might be taken into account, thereby improving the fix accuracy.

\section{Ray Resolution by Direction-of-Arrival}

When the component rays of a multimode signal arrive from directions which differ sufficiently, they can be resolved by a directive antenna system and displayed separately. The Wullenweber direction- finding system at the University of Illinois is such a svstem. It consists of an arrav of 120 vertical antenna elements (folded monopoles) disposed on a circle 994 feet in diameter on the outside of a reflecting wire screen 955 feet in diameter. The elements are used 40 at a time, those in use being coupled to the receiver through a phasing system which forms a fan-shaped radiation pattern with a small azimuthal beamwidth. A rotary capacitive switch is provided to scan the directive pattern in azimuth by successively connecting a new element at one end of the set of 40, while dropping one at the other end. The capacitive switch is motor driven at a rate of 900 rpm. Thus a complete azimuth scan is obtained in $1 / 15$ sec. The switch can also be manually set.

The output signal from the receiver is visually displayed, causing a radial deflection of the spot on a cathode ray tube whose deflection yoke rotates in sychronism with the scanning switch.

The system is designed to operate in the frequency range from 4 to $16 \mathrm{Mc} / \mathrm{s}$. The half-power beamwidth ranges from $14^{\circ}$ at $4 \mathrm{Mc} / \mathrm{s}$ to $3^{\circ}$ at $16 \mathrm{Mc} / \mathrm{s}$.

The signals from radio station WWV are particularly convenient for study because of their simplicity and their continuous presence on several frequencies, and because of the favorable transmitter location with respect to the receiving site. The direct path length is about $1000 \mathrm{~km}$. The variety of propagation phenomena whose directional characteristics are observable is gratifying, and not a little surprising. The system has been particularly successful in conveying a description of the angular spectrum of signals which are too complex to be handled on smaller direction finding systems. Some examples of such signals are described in the following paragraphs. In interpreting the figures, note that the great circle bearing of the transmitter is $93^{\circ}$.

One of the interesting phenomena observed is the nature of the signal breakup as the MUF drops down through the operating frequency. This is presently observable on the $10 \mathrm{Mc} / \mathrm{s}$ transmissions of $\mathrm{WWV}$ almost every evening. Figure 14 shows a sequence of photographs of the azimuth display taken during an afternoon and evening. The first in this series shows the nature of the display on a single-ray signal. It is essentially a polar plot of the array radiation pattern for this frequency $(10 \mathrm{Mc} / \mathrm{s})$. The irregularity in the nose of the pattern is a commutation defect. Multiple ray arrivals at angles appreciably off the great circle bearing are evident in the intermediate photographs. Multiray character can be observed even where the rays are not resolved by the broadening of the response pattern. In the last photograph only scatter signal is present. It is, however, appreciably above the noise level. While the details of the signal behavior vary from evening to evening, the general pattern is usually similar to that shown here.

In figure 15 are shown two examples of fairly strong, but highly non-specular incident signals. They are from $\mathrm{WWV}$ on $10 \mathrm{Mc} / \mathrm{s}$. Such signals are characterized by very high fading rates (up to 20 or 30 fades per second). 
a
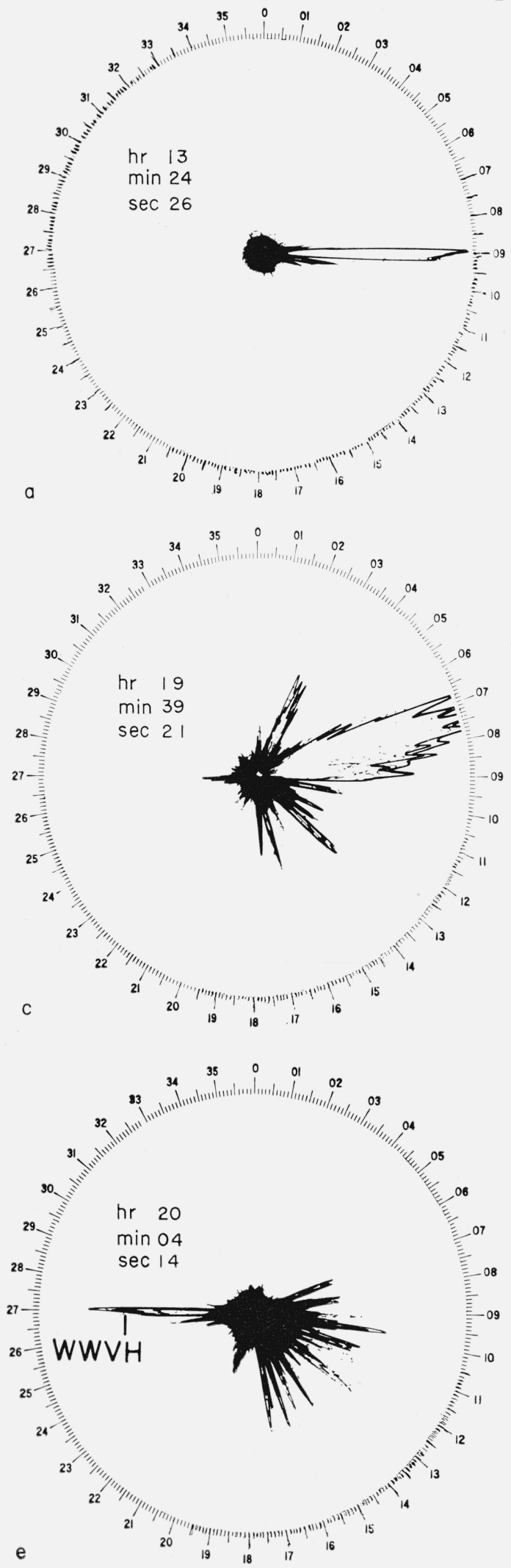
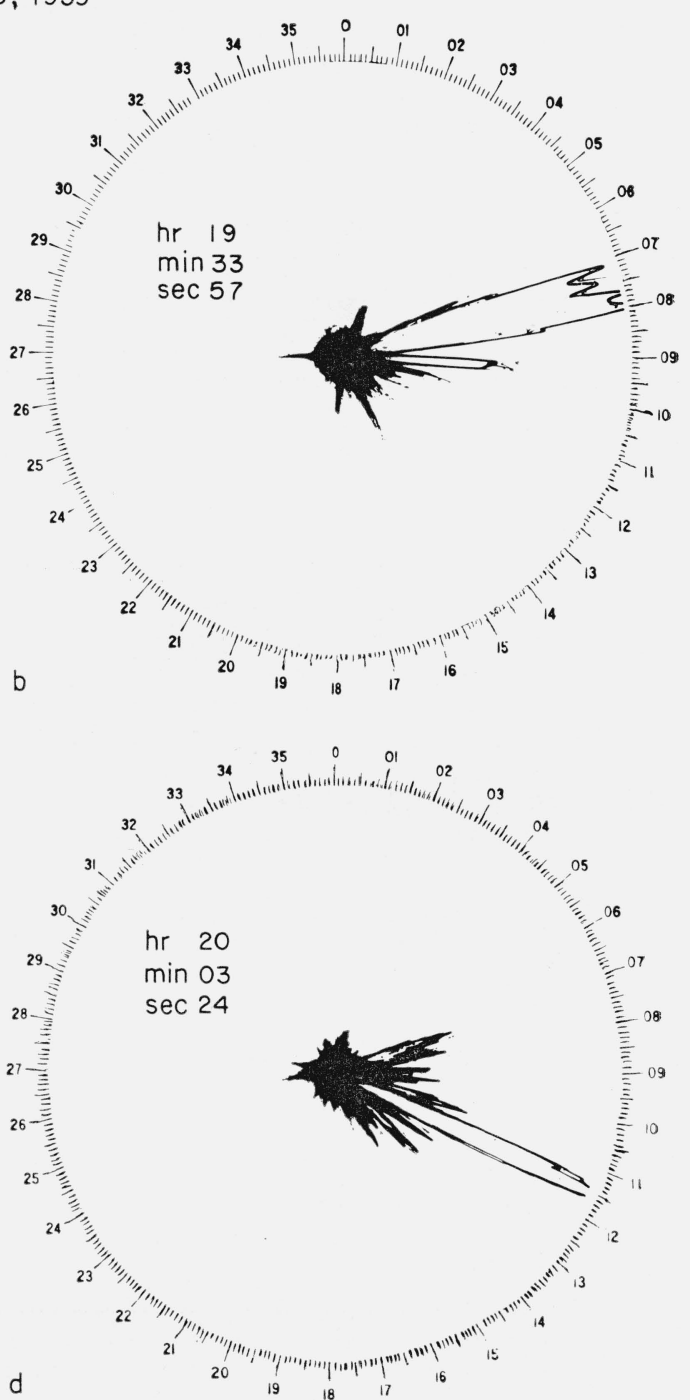

Figure 14. Sequence of signal displays showing signal breakup as MUF decreases, passing through operating frequency; signal is from $W W V$ on $10 \mathrm{Mc} / \mathrm{s}$, great circle bearing is $93^{\circ}$. 

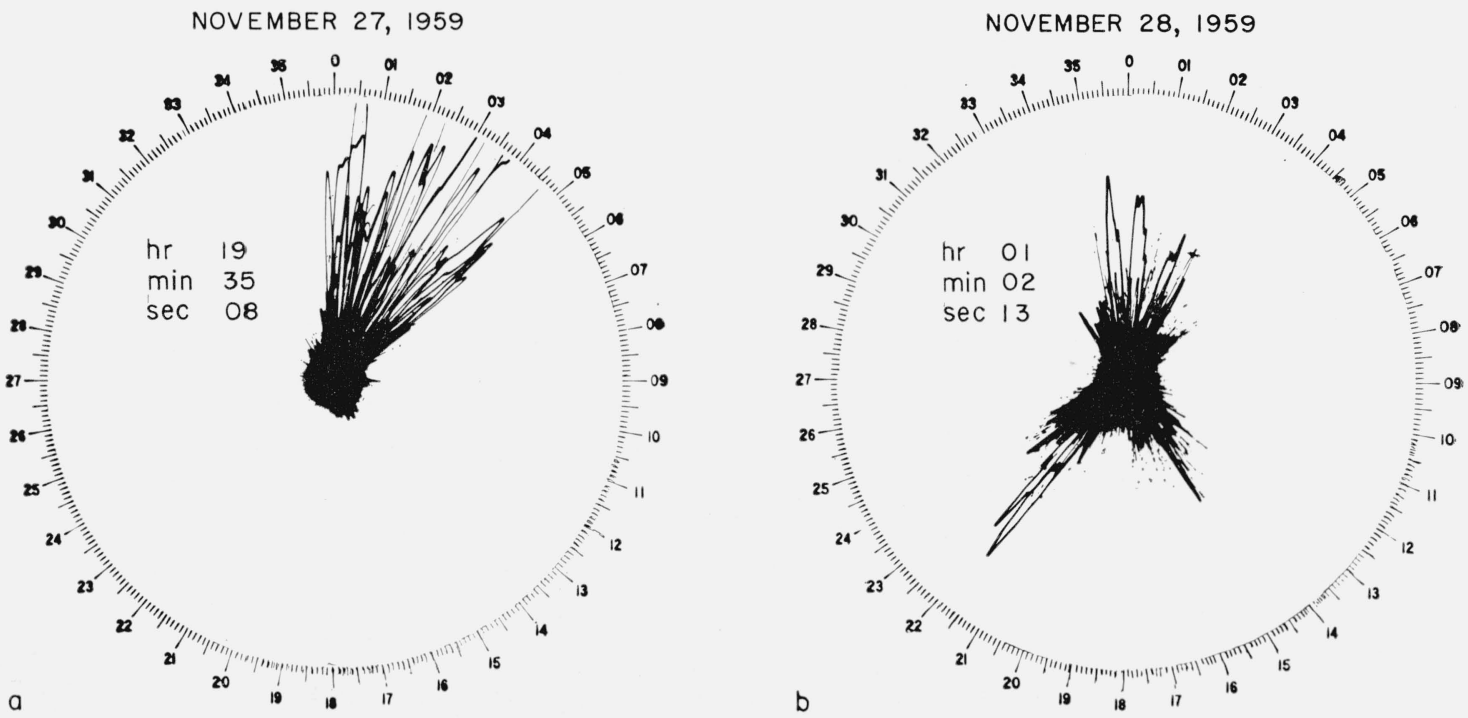

Figure 15. Examples of anomalous signals propagated via spread-F and scatter structures.

a, Spread- $F$ signal; b, Scatter signal.

Signals of the type shown in figure 15 a seem to be associated with the existence of spread- $F$ over eastern Canada. Such displays are frequently observable, and may last from a few minutes to several hours. Energy may be incident over an angular sector ranging from $5^{\circ}$ to $60^{\circ}$.

Using some reasonable assumptions, it has been possible to estimate the point of reflection for some signals of this type. It is often possible to receive a normal on-path signal from WWV on $5 \mathrm{Mc} / \mathrm{s}$ at times when anomalous displays are observed on $10 \mathrm{Mc} / \mathrm{s}$. By setting the steerable beam manually, it has been possible to measure, as a function of direction of ray arrival, the difference in time of flight between the time ticks propagated in a regular mode and that of the ticks propagated in the anomalous mode. Assuming that the anomalous reflection occurs at $F$-region heights, knowledge of the direction of arrival and excess time of flight permits location of the reflection point. A number of such determinations are shown in figure 16. Each series of points connected by a line represents a number of measurements on one display of the phenomenon. A period of the order of 20 minutes was required to obtain the measurements in each set. The different sets were measured on different occurrences of the phenomenon. A number of parallels of geomagnetic latitude have been added to the map for reference to show the tendency of the reflection point loci to lie, at any one time, along parallels of geomagnetic latitude.

Figure $15 \mathrm{~b}$ shows another type of non-specular signal having even less regularity and higher fading rate than that of figure $15 \mathrm{a}$. Such signals have been called "scatter" signals. There are times when the individual raylet behavior in these signals is suggestive of reflection from dense meteor trails.
Attempts have been made to plot reflection point loci for signals of this sort. However, because of the very rapidly changing configuration of the display, these attempts have met with much less success than was achieved on signals such as shown in figure $15 \mathrm{a}$.

All of these phenomena are being made subjects for study in greater detail.

\section{Conclusion}

The two experimental studies described demonstrate the power of direction-finding techniques in studying propagation phenomena. A particularly promising extension of this work is the tracking of a frequency-swept ionosonde with high-resolution DF equipment. An experiment of this type is being planned.

The experiments described above were performed by members of the staff of the Radio DirectionFinding Research Laboratory, Department of Electrical Engineering, University of Illinois, as parts of research programs sponsored by the Office of Naval Research and the Bureau of Ships.

\section{Bibliography}

Bailey, A. D., An investigation of the direction of arrival of radio waves, Thesis submitted in partial fulfillment of the requirements for the degree of Doctor of Philosophy, University of Illinois, Urbana, Ill. (1954).

Bain, W. C., The calculation of wave-interference errors on a direction-finder employing cyclical differential measurement of phase, Proc. Inst. Elec. Eng. 100, Pt. III, pp. 253-261 (September 1953). 


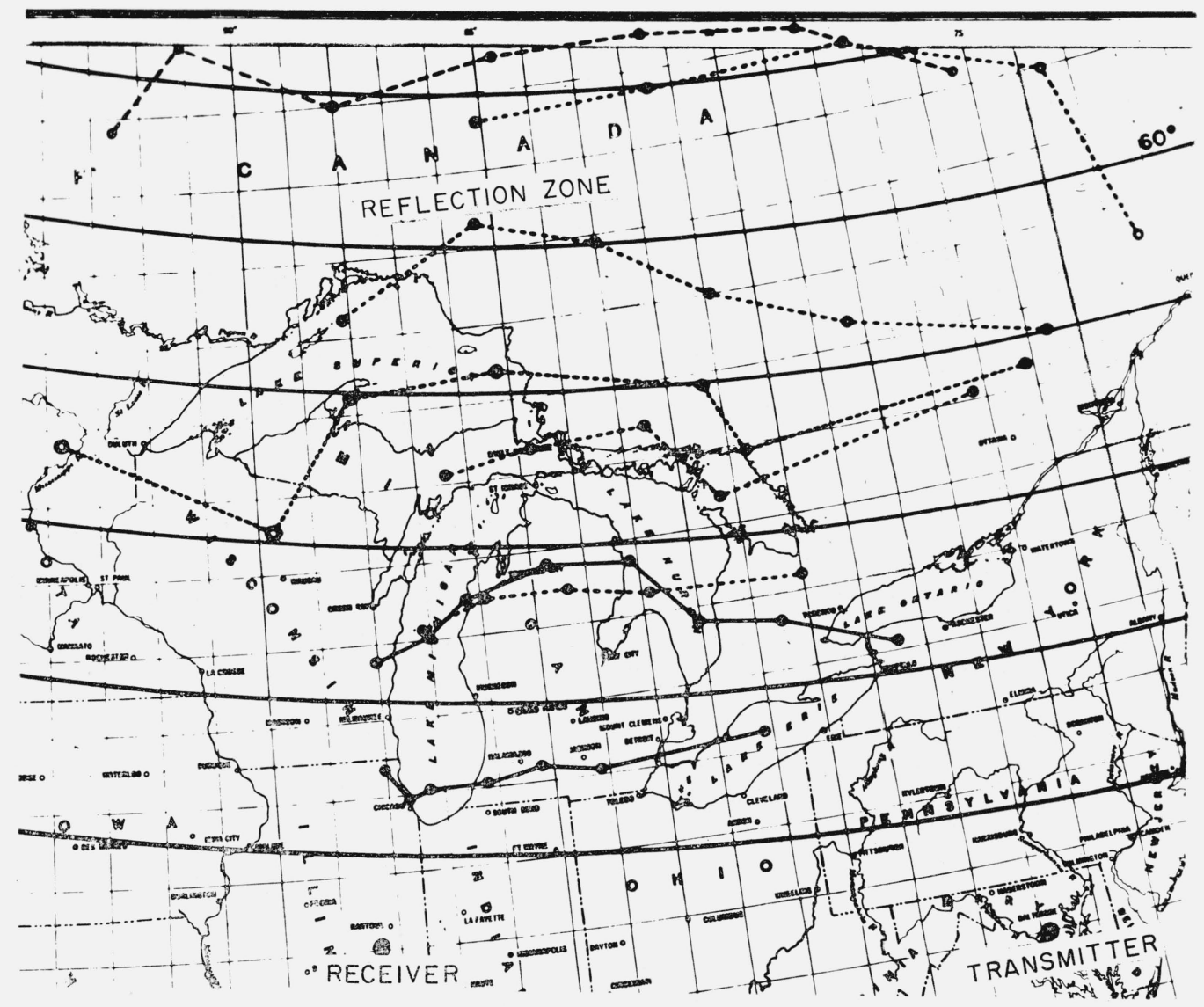

FiguRE 16. Estimated reflection points for spread-F signals.

Bain, W. C., On the rapidity of fluctuations in continuouswave radio bearings at high frequencies, Proc. Inst. Elec. Eng., 102, Pt. B, pp. 541-543 (July 1955).

Bain, W. C., The theoretical design of direction-finding systems for high frequencies, Proc. Inst. Elec. Eng. 103, pt. B, pp. 113-119 (January 1956).

Bain, W. C., Possible errors of a particular wide-aperture direction-finder, Proc. Inst. Elec. Eng., Mono. No. 170R, pp. 1-12 (March 1956).

Bain, W. C., Fluctuations in continuous-wave radio bearings at high frequencies. Proc. Inst. Elec. Eng. 103, p. 560 (July 1956).

Barfield, R. H. and W. Ross, The measurement of lateral deviation of radio waves by means of a spaced loop direction finder, J. Inst. Elec. Eng. 83, p. 98 (1938).

Bramley, E. N., Some aspects of the rapid directional fluctuations of short radio waves reflected at the ionosphere, Proc. Inst. Elec. Eng. 102, pt. B, pp. 533-540 (July 1955).

Barmley, E. N., Some comparative directional measurements on short radio waves over different transmission paths, Proc. Inst. Elec. Eng. 102, pt. B., pp. 544-549 (July 1955).

Bramley, E. N., H. F. bearing variations of an Adcock Direction-Finder, Proc. Inst. Elec. Eng., Mono. No. 175R, pp. 1-7 (April 1956).

Clemmow, P. C. and R. F. Mullaly, The dependence of the refractive index in magneto-ionic theory on the direction of the wave normal, The Physics of the Ionosphere, Report of the Physical Society Conference on the physis of the ionosphere held at the Cavendish Laboratory, September, 1954, The Physical Society (London, 1955).
Gleason, R. F. and J. H. Trexler, Ionospheric limitations in the ultimate accuracy of direction finding, NRL Memorandum Report No. 61, Naval Research Laboratory (Washington, D.C., 1952).

Heiligtag, Th., Uber die Grunde der Missweisungen beim Richtungempfang, Jahrbuch Zeitschrift fur Drahtlose Telegraphie and Telephonie 21, p. 77 (1923).

Jordan, E. C., et al. Summary technical report, Technica Report No. 4, The Radio Direction Finding Research Laboratory, Department of Electrical Engineering, University of Illinois, Urbana, Ill.

Ross, W., Lateral deviations of radio waves reflected at the ionosphere, Department of Scientific and Industrial Research, Radio Research, Special Rept. No. 19, H. M. Stationery Office (London, 1949).

Ross, W., Fundamental problems in radio direction finding at high frequencies, J. Inst. Elec. Eng., pt. IIIA 94, pp. 154-165 (1947)

Smith, S. B., and H. G. Hopkins, H.F. direction finding, Wireless Engineer, 31, pp. 11-14 (January 1954).

Fundamental Principles of Ionospheric Transmission, Department of Scientific and Industrial Research, Radio Research, Special Rept. No. 17, H. M. Stationery Office (London, 1948).

Ionospheric Radio Propagation, NBS Circ. 462 (1948).

(Paper 65D3-120) 